\title{
Upregulation of centromere protein $M$ promotes tumorigenesis: A potential predictive target for cancer in humans
}

\author{
YING LIU ${ }^{1}$, WENFENG YU ${ }^{1}$, PENG REN $^{2}$ and TING ZHANG ${ }^{1}$ \\ ${ }^{1}$ Key Laboratory of Endemic and Ethnic Diseases of The Ministry of Education, Guizhou Medical University, \\ Guiyang, Guizhou 550004; ${ }^{2}$ Department of Urology, The Second Affiliated Hospital of Guizhou Medical University, \\ Guiyang, Guizhou 556000, P.R. China
}

Received January 10, 2020; Accepted July 20, 2020

DOI: $10.3892 / \mathrm{mmr} .2020 .11461$

\begin{abstract}
Centromere protein M (CENPM), a protein required for chromosome separation, is involved in in mitosis. However, little has been reported about the roles of CENPM in various types of cancer. The present study identified that the mRNA expression levels of CENPM were significantly upregulated in 14 types of human cancer and identified a positive association between CENPM mRNA expression and patient mortality using the Oncomine, Gene Expression Profiling Interactive Analysis, Human Protein Atlas and Kaplan-Meier Plotter databases. A protein interaction network constructed with CENPM-interacting genes obtained from the cBioPortal demonstrated that nine genes participating in the cell cycle served key roles in the function of CENPM. Cell cycle analysis, reverse transcription-quantitative polymerase chain reaction, a Cell Counting Kit-8-based proliferation assay and a terminal deoxynucleotidyl transferase dUTP nick end labelling assay further revealed the tumorigenic and carcinogenic roles of CENPM in vitro. In addition, it was identified that the mRNA expression levels of five of the nine identified genes were significantly associated with CENPM in MCF7 cells and that CENPM was rarely mutated among various types of human cancer. In conclusion, the data from the present study revealed that CENPM exerted its pro-tumorigenic function by regulating cell cycle-associated protein expression and
\end{abstract}

Correspondence to: Dr Ying Liu or Dr Wenfeng Yu, Key Laboratory of Endemic and Ethnic Diseases of The Ministry of Education, Guizhou Medical University, 9 Beijing Road, Guiyang, Guizhou 550004, P.R. China

E-mail: 315324940@qq.com

E-mail: wenfengyu3222@yandex.com

Abbreviations: CENPM, centromere protein $\mathrm{M}$; $\mathrm{BC}$, breast cancer; TCGA, The Cancer Genome Atlas; BRCA, breast invasive carcinoma; CESC, cervical squamous cell carcinoma and endocervical adenocarcinoma; LIHC, liver hepatocellular carcinoma; SARC, sarcoma; DLBC, diffuse large B-cell lymphoma; LUAD, lung carcinoma; LUSC, lung squamous cell carcinoma

Key words: centromere protein M, cell cycle, breast cancer suggested that CENPM could be used as a prognostic marker for breast cancer.

\section{Introduction}

Breast cancer $(\mathrm{BC})$ is the most common cancer occurring in women, and $\sim 271,270$ patients were newly diagnosed with BC in 2019 in the United States (1). According to the National Center for Health Statistics (NCHS), the mortality rate of $\mathrm{BC}$ in 2012-2016 was 20.6\%, which ranked BC as the second most life-threatening disease in the United States following lung/bronchus cancer. In addition, the NCHS estimated that BC would cause $\sim 42,260$ deaths in 2019 (1). BC is typically categorized as basal-like [estrogen receptor low/progesterone receptor low/human epidermal growth factor receptor 2 (HER2) low], luminal A-type (estrogen receptor high/HER2 low), luminal B-type (estrogen receptor or progesterone receptor high, either HER2 high or low, and Ki67 high) or HER2-type (estrogen receptor low/progesterone receptor low/HER2 high) based on the cell types of origin. Notably, the incidence of luminal A-type is the highest among all types of $\mathrm{BC}$ (2). As numerous individuals are likely to be affected by $\mathrm{BC}$ over the coming years, elucidating the mechanisms underlying $\mathrm{BC}$ to identify a novel prognostic marker could be important for BC therapy.

Cancer cells regularly exhibit altered chromosome numbers; these alterations are collectively known as aneuploidy $(3,4)$. Aneuploidy, which is usually caused by an abnormal number and size of the centrosome, may accelerate tumorigenesis and carcinogenesis by causing chromosomes to separate unequally during mitosis (4). It has been reported that $65-90 \%$ of BC cells exhibit aneuploidy (3). Centromere protein M (CENPM) is an essential centromere component that is associated with several other centromere proteins [including CENPA (5), CENPC (6), CENPI (7) and CENPH (8)], and is required for chromosome separation. The CENPM protein interacts with other proteins to form a vital complex that preserves kinetochore and spindle microtubule attachment during metaphase (9). Overexpression of CENPM leads to unequal numbers of chromosomes in cells; these cells subsequently exit mitosis, survive and lead to aneuploidy (10). In addition, high CENPM expression has been reported to be associated with primary melanoma (11), bladder cancer (12), hepatocellular carcinoma (13), and head and neck 
squamous cell carcinoma (14). Based on these studies, the present study hypothesized that aberrant CENPM could function as an oncogene by intervening in the progression of the cell cycle.

The present study demonstrated that CENPM mRNA expression was upregulated in various types of cancer and that elevated CENPM in BC was highly associated with low patient survival probability. By studying CENPM-related genes, it was identified that five key genes associated with CENPM could accelerate the cell cycle in BC. In addition, in MCF7 cells, CENPM overexpression significantly enhanced cell proliferation and inhibited apoptosis in vitro. Therefore, the findings of the present study indicated that CENPM may control tumor progression and survival rate in patients with $\mathrm{BC}$.

\section{Materials and methods}

Catalogue of Somatic Mutations in Cancer (COSMIC) database analysis. The COSMIC (https://cancer.sanger. ac.uk/cosmic) is a database of gene mutations associated with various types of human cancer, which includes 1,420,135 samples and 26,878 papers (COSMIC v89). Using this database, information about CENPM gene mutational signatures according to cancer types was extracted, and the association between CENPM mutations, including point mutations, insertions and deletions, and the risk of $\mathrm{BC}$ tumorigenesis was summarized. Detailed information about the mutational signature method is provided in previous studies $(15,16)$.

Oncomine database analysis. Oncomine v4.5 (https://www. oncomine.org/resource/login.html) is a database of information levels, DNA copy numbers, drug sensitivity and other parameters in normal samples and multiple types of cancer samples. After a target gene is selected, the Oncomine database returns a list of gene expression analyses that have been conducted in different microarray studies, allowing easy exploration of the diseases that are possibly caused by the selected gene. In the present study, the analyses revealing elevated CENPM expression were acquired to create a graph showing the cancer types that are significantly associated with CENPM gene expression. The detailed methods used for Oncomine database analysis are described in a previous study (17). Subsequently, Gene Expression Profiling Interactive Analysis (GEPIA) v1 (http://gepia.cancer-pku.cn/) was applied to reveal CENPM mRNA expression levels in several types of cancer samples vs. normal samples. The detailed methods used for GEPIA are described in a previous study (18).

cBioPortal database analysis. cBioPortal v3.4.4 (http://www. cbioportal.org/) is a database of information on gene expression, genetic alterations, coexpression and survival status that enables researchers to analyze and explore topics associated with tumorigenesis. Upon selection of invasive breast carcinoma [The Cancer Genome Atlas (TCGA), cell 2015] (19), and the gene of interest (CENPM), the database returned a list of genes highly associated with CENPM. To visualize the protein-protein interaction network, the Search Tool for the Retrieval of Interacting Genes/proteins (STRING) database v11.0 (https://string-db.org/) was accessed with the gene names provided by the cBioPortal database, the nodes and lines were modified with Cytoscape (CytoHubba) v3.42 (https://cytoscape.org/), and protein expression was examined by using the Human Protein Atlas v19 (HPA; www.proteinatlas.org). GeneCards Version 4.11 (https://www.genecards.org/) was used to search for the functional information of the candidate genes. The detailed methods used for gene coexpression analysis are described in previous studies $(20,21)$.

Kaplan-Meier plotter analysis. The UALCAN database (September 23, 2019 release; http://ualcan.path.uab.edu), a user-friendly platform that links to cancer omics databases TCGA and MET500 (September 11,2017 release; https://met500. path.med.umich.edu/), can be used to evaluate gene expression vs. patient survival probability or tumor grade for multiple types of cancer. To calculate the effect of the target gene expression on patient survival, UALCAN was used to create a Kaplan-Meier plot with sample data by using the 'survival' and 'survminer' packages. The detailed methods are provided in a previous study (22). The association between CENPM mRNA upregulation and patient survival status in various types of cancer were identified. To estimate the prognostic value of CENPM in specific BC types, including luminal A-type, luminal B-type, HER2-positive-type and basal-like-type BC, CENPM (Gene ID, 218741_at, JetSet best probe set) was queried on the Kaplan-Meier Plotter database (August 1, 2019 release; http://www.kmplot.com) that links to Gene Expression Omnibus, European Genome-Phenome Archive and TCGA data, and the results clearly demonstrated the potential effects of high or low levels of CENPM on patient survival. The survival curves of samples with high gene expression and low/medium gene expression were compared by log rank test. The detailed methods are provided in a previous study (23).

Reverse transcription-quantitative (RT-q)PCR. MCF7 cells (American Type Culture Collection) were cultured in DMEM medium (Sigma-Aldrich; Merck KGaA) containing 10\% fetal bovine serum (FBS; Gibco; Thermo Fisher Scientific, Inc.), then received treatment in serum-free DMEM medium. Cellular mRNA was extracted and purified from MCF7 cells $\left(1 \times 10^{6} / \mathrm{ml}\right)$ using TRIzol ${ }^{\circledR}$ (Invitrogen; Thermo Fisher Scientific, Inc.), chloroform, isopropanol and alcohol. The cDNA was synthesized by using RevertAid RT Reverse Transcription Kit (Thermo Fisher Scientific, Inc.), in accordance with the manufacturer's protocols. Real time PCR was performed with ABI PRISM 7300 Sequence Detection System (Applied Biosystems, USA). After RT, the target gene mRNA expression was detected with the primers shown in Table I and with FastStart Universal SYBR Green Master (Rox; Roche Molecular Systems, Inc.), in accordance with the manufacturer's protocols. The thermal cycling conditions were: Denaturation $10 \mathrm{~min}$ at $95^{\circ} \mathrm{C}$, followed by 45 cycles of denaturation at $95^{\circ} \mathrm{C}$ for $30 \mathrm{sec}$, annealing at $56^{\circ} \mathrm{C}$ for $30 \mathrm{sec}$ and extension at $72^{\circ} \mathrm{C}$ for $30 \mathrm{sec}$, and then CENPM, ERCC6L, SHCBP1, CKS2, RAD51, KIF4A, HMMR, SPAG5, CDC25C, and RACGAP1 mRNA levels were quantified as previously reported (24).

Western blot analysis. Briefly, after protein was extracted from MCF7 cells with RIPA buffer according to the manufacture's protocol (Cell Signaling Technology, Inc.), the concentration of protein was determined by Bradford 
Table I. Primers used for reverse transcription-quantitative PCR.

\begin{tabular}{lll}
\hline Gene & \multicolumn{1}{c}{ Forward (5'-3') } & \multicolumn{1}{c}{ Reverse (5'-3') } \\
\hline$P C N A$ & CCTGCTGGGATATTAGCTCCA & CAGACCCATTTACTTGTGTTGGA \\
GAPDH & ACAACTTTGGTATCGTGGAAGG & GCCATCACGCCACAGTTTC \\
$C E N P \mathrm{~N} M$ & ACGCCTGGTTACTATCAAAAGG & CAGCGGTAGGTGTCGAAGC \\
$E R C C 6 L$ & GCGGACTCGATGCTCAAAGA & TTCTGGAGACTGTATTTGCTGTG \\
$S H C B P 1$ & CTCTGGCTTGCTACTTTATCGAG & TGCATCAAACATACCGGAAAGG \\
$C K S 2$ & GCTACCGTGATAAACCAGGTTC & AGGCTCTGAATCGCTCATAGA \\
$R A D 51$ & TTCGACGAACACTACGAGTACC & GGACACCAAGTCTCCTCCAC \\
$K I F 4 A$ & CAACCCATTTCACGGTTAGAGC & TTCTTTGGCGCATAGGCAACA \\
$H M M R$ & TACTGCGGTGGAGCAAGAAG & CATCTGCGCTTGACGGAGAG \\
$S P A G 5$ & ATGATGGCTAAGCAAGAAGGC & TTTCCCTTGAGACTCTTCGAGA \\
$C D C 25 C$ & TTGAGGCCCGTTTAGATACCA & GCTTTCCTTGGAGCAATGTAGTT \\
$R A C G A P$ & TCTACGGAACTCTTCTCATCCAC & TCCAGGAGCAGGTTTAACATTTT \\
& ATGATGCTGAATGTGCGGAAT & CGCCAACTGGATAAATTGGACTT
\end{tabular}

protein assays kit (Beyotime Institute of Biotechnology) according to the manufacturer's protocols, and $40 \mu \mathrm{g}$ proteins were loaded into each lane of a 12 or $15 \%$ SDS-PAGE gel. Following gel electrophoresis and transfer of the separated proteins onto a nitrocellulose membrane, the membrane was treated with $10 \mathrm{ml}$ blocking buffer (Beyotime Institute of Biotechnology, Inc.) containing 5\% BSA for $1 \mathrm{~h}$ at room temperature. Subsequently, the membrane was incubated with anti-cleaved caspase-3 (cat. no. 9661; 1:1,000; Cell Signaling Technology, Inc.), anti-Bax (cat. no. 5023; 1:1,000; Cell Signaling Technology, Inc.), anti-Bcl-2 (cat. no. 15071; 1:1,000; Cell Signaling Technology, Inc.), anti-cyclin-dependent kinase subunit 2 (CKS2; cat. no. ab155078; 1:1,000; Abcam, Inc.), anti-GAPDH (cat. no. 5174; 1:1,000; Cell Signaling Technology, Inc.) or anti- $\beta$-actin (cat. no. 4970; 1:1,000; Cell Signaling Technology, Inc.) primary antibodies for $12 \mathrm{~h}$ at $4^{\circ} \mathrm{C}$. Following incubation with anti-rabbit secondary antibodies (cat. no. 7074; 1:5,000; Cell Signaling Technology, Inc.), or anti-mouse secondary antibodies (cat. no. 7076; 1:5,000; Cell Signaling Technology, Inc.) for $1 \mathrm{~h}$ at room temperature. Protein bands were detected with Electro-chemiluminescence (ECL) Plus reagents (Cytiva), according to the manufacturer's instructions, the membrane was imaged with a GeneGnome XRQ Chemiluminescence Imaging system (Gene Company Ltd.), and the density was quantified with ImageJ software (v1.46; National Institutes of Health).

Cell cycle analysis. To construct CENPM-overexpression vectors, CENPM cDNA was amplified by polymerase chain reaction from the MCF7 cells with the following primers: Forward, 5'-CATGCTAGCATGTCGGTGTTGAGGCCC CTGGA-3'; reverse: 5'-TTCAAGCTTTCACAGGTCCTC CAGGGAGGGGC-3'. The PCR product was digested with NheI and HindIII, and then subcloned into an expression vector pIRES2-EGFP (pIRES2-EGFP, Clontech Laboratories, Inc.). The plasmid was termed the CENPM-overexpression vectors, and then CENPM-overexpression vectors at a final concentration $0,1.5$ or $2 \mu \mathrm{M}$ with or without control vector (pIRES2-EGFP) were transfected into MCF7 cells $\left(1 \times 10^{6}\right)$ using the Lipofectamine ${ }^{\circledR} 2000$ Reagent (Invitrogen; Thermo Fisher Scientific, Inc.), following the manufacturer's protocol. MCF7 cells were transfected for $12 \mathrm{~h}$, grown in DMEM medium supplemented with $5 \%$ fetal bovine serum (FBS; Gibco; Thermo Fisher Scientific, Inc.) for $24 \mathrm{~h}$ at $37^{\circ} \mathrm{C}$ and then fixed in $70 \%$ ethanol for $12 \mathrm{~h}$ at $4^{\circ} \mathrm{C}$. The ethanol-fixed samples were washed twice with PBS, digested with RNase A $\left(1 \mathrm{mg} / \mathrm{ml}\right.$; Sigma-Aldrich; Merck KGaA) for $30 \mathrm{~min}$ at $37^{\circ} \mathrm{C}$, and then stained with propidium iodide solution $(1 \mathrm{mg} / \mathrm{ml}$; Sigma-Aldrich; Merck KGaA) for $30 \mathrm{~min}$ at $4^{\circ} \mathrm{C}$. The cell cycle was examined by a BD FACSVerse flow cytometer (BD Biosciences, San Jose, CA) at an excitation wavelength of $488 \mathrm{~nm}$ and the data were analyzed with ModFit LT v4.0.5 Software (Verity Software House, Inc.).

Cell Counting Kit-8 (CCK-8)-based proliferation assay. CCK-8 assay was conducted to assess proliferation at $0,12,24$ and $48 \mathrm{~h}$ and T-47D cells (American Type Culture Collection) were cultured in RPMI-1640 medium (Sigma-Aldrich) containing $10 \%$ fetal bovine serum at $37^{\circ} \mathrm{C}$ under a $5 \%$ $\mathrm{CO}_{2}$ atmosphere. Following transfection, the MCF7 and T-47D cells were passaged at a density of 2,000 cells/well in 96-well plates, cells in each well were incubated with $10 \mu 1$ CCK-8 solution (MedChemExpress) for $2 \mathrm{~h}$ at $37^{\circ} \mathrm{C}$, and the proliferation rate was calculated based on the absorbance at $450 \mathrm{~nm}$, as determined using a microplate reader (ELx800, BioTek Instruments).

Terminal deoxynucleotidyl transferase dUTP nick end labeling (TUNEL) assay. For determination of apoptosis, control- or CENPM-overexpressing MCF7 cells were stained using the One Step TUNEL Apoptosis Assay Kit (Beyotime Institute of Biotechnology), as described in the manufacturer's protocols, and immunofluorescence was determined by fluorescence microscopy (Leica Microsystems $\mathrm{GmbH}$ ), with 5 randomly selected high-power fields to calculate the ratio per slide.

Cell scratch assay. MCF7 cells ( $\sim 90 \%$ confluence) were transfected with control- or CENPM-overexpression vectors 
for $12 \mathrm{~h}$, scratched with $200 \mu \mathrm{l}$ pipette tips, washed with PBS, and grown in medium supplemented with 1 or $5 \%$ FBS for 0 and $48 \mathrm{~h}$ (25). Following the indicated culture times, the gaps were imaged with a fluorescence microscope (Leica Microsystems $\mathrm{GmbH}$ ) and the width of each gap was analyzed with ImageJ (v1.46; National Institutes of Health).

RNA interference. Three CENPM-targeting short hairpin (sh)RNAs were constructed; the sequences were as follows: shCEN \#a, 5'-CCTGATCGTGTTTGTGGTTAA-3'; shCEN \#b, 5'-GCTGACTCCATAAACATTCTC-3'; shCEN \#c, 5'-GCG GACTCGATGCTCAAAGAG-3'. The shRNAs specifically targeting human CENPM were cloned into psi-LVRU6GP (GeneCopoeia,Inc.) and the non-targeting sequence 5'-ACAGAA GCGATTGTTGATC-3' was cloned into the same vector and used as the shControl. A Lenti-Pac HIV Expression Packaging kit (GeneCopoeia, Inc.) was used for shRNA-encoding lentivirus packaging, according to the manufacturer's protocols. The detailed methods used for lentivirus infection and confirmation of CENPM knockdown are provided in a previous study (26). Briefly, 293T cells ( $70 \%$ confluence) were seeded in 6 well plate for $24 \mathrm{~h}$, transfected with $500 \mathrm{ng}$ shControl, shCEN \#a, shCEN \#b, or shCEN \#c, 250 ng pMD2.G, 250 ng psPAX2, and Lipofectamine ${ }^{\circledR} 2000$ Reagent (Invitrogen; Thermo Fisher Scientific, Inc.) for $24 \mathrm{~h}$, then the medium was refreshed using freshly prepared 5\% DMEM. At $72 \mathrm{~h}$ after transfection, the supernatants were harvested, the $400 \mu \mathrm{l}$ virus solution was added to the cells and the knockdown of the target gene was confirmed by real time PCR.

Colony formation test. Transfected cells were plated in 6-well plates with 1,000 cells per well and then cultured for 10 days until colonies were visible to the eye. Cells were washed twice with phosphate-buffered saline (PBS), fixed with methanol for $30 \mathrm{~min}$ at room temperature, and then stained with crystal violet for $30 \mathrm{~min}$ at room temperature. The number of colonies was counted.

Statistical analysis. All experiments were conducted at least three times independently, and the data were analyzed by two-tailed, unpaired Student's t-test or one-way ANOVA followed by Tukey's or Dunnett's multiple comparisons test. The data are presented as the means \pm standard error of the mean and $\mathrm{P}<0.05$ was considered to indicate a statistically significant difference. The survival curves of samples with high gene expression and low/medium gene expression were compared by log rank test. Data were analyzed using GraphPad v5.01 (GraphPad Software, Inc.).

\section{Results}

Expression levels of CENPM in various types of human cancers. By using the Oncomine database, the transcription levels of CENPM in normal and cancerous human specimens were examined (Fig. 1A). CENPM mRNA was significantly upregulated in 14 of 20 types of cancer (Fig. 1A, fold change $>2$, $\mathrm{P}<1 \times 10^{-4}$, gene rank: Top 10\%). The 43 datasets associated with elevated CENPM mRNA expression included 4,697 samples, and the fold changes in expression ranged between 2.008 and 9.628. CENPM mRNA upregulation appeared most often in BC (22.2\%), cervical cancer (40\%), liver cancer $(23 \%)$, lung cancer (22.2\%), lymphoma (16\%) and sarcoma (16\%) (Fig. 1B). Subsequently, the mRNA expression levels of CENPM were analyzed using an online database (GEPIA) (18) to evaluate the effect of CENPM on human cancer. The results demonstrated that breast invasive carcinoma (BRCA), cervical squamous cell carcinoma and endocervical adenocarcinoma (CESC), liver hepatocellular carcinoma (LIHC), sarcoma (SARC), diffuse large B-cell lymphoma (DLBC), lung adenocarcinoma (LUAD) and lung squamous cell carcinoma (LUSC) tissues had significantly elevated levels of CENPM mRNA (Fig. 1C and D).

The protein expression levels of CENPM were detected using the Human Protein Atlas (HPA; www.proteinatlas.org). Since immunohistochemical (IHC) staining data for CENPM in SARC or DLBC were not available in the HPA database, the protein expression levels of CENPM in SARC or DLBC were not investigated. As shown in Fig. 2A, the protein expression levels of CENPM were slightly elevated in BRCA $(n=2$ for normal breast tissue and $n=2$ for BRCA tissue) and CESC $(n=2$ for normal cervical tissue and $n=2$ for CESC tissue), and no significant difference was observed in LIHC $(n=3$ for normal liver tissue and $\mathrm{n}=1$ for LIHC tissue; $\mathrm{P}>0.05$ ) and LUSC ( $n=2$ for normal lung tissue and $n=4$ for LUSC tissue). However, it is highly recommended that further IHC studies be performed on normal and cancerous samples to verify these findings. Furthermore, the IHC staining assay illustrated that the CENPM protein was primarily expressed in the nuclei of cells and its localization was associated with its function (9).

Next, the association of CENPM gene expression with various types of human cancer was examined, and the association between CENPM mRNA expression and cancer grade was further examined using the GEPIA database. As shown in Fig. 2B, elevated CENPM mRNA expression was positively associated with tumor grade in BRCA, LIHC and LUAD, but barely in CESC and DLBC (data not shown). The comparatively few specimens from patients with SARC (Fig. 1D, $n=2$ for normal tissue) made the results of the analysis of the association of CENPM gene expression with tumor grade in SARC less meaningful (data not shown). Next, it was examined whether CENPM gene expression in BRCA, CESC, LIHC, SARC, DLBC, LUAD and LUSC was associated with overall survival rates using the online UALCAN databases (22). As shown in Fig. 2C, lower survival rates in BRCA (881 patients, $\mathrm{P}=0.021$ ), LIHC (365 patients, $\mathrm{P}=0.00027$ ) and LUAD (502 patients, $\mathrm{P}=0.0011)$ were associated with higher CENPM mRNA levels. By contrast, elevated CENPM mRNA levels were associated with higher survival rates in patients with CESC (296 patients, $\mathrm{P}=0.00014)$ but were not associated with survival rates in SARC (259 patients, $\mathrm{P}=0.29$ ), $\mathrm{DLBC}$ (47 patients, $\mathrm{P}=0.039$; too few patients to confirm) or LUSC (494 patients, $\mathrm{P}=0.25$ ) (data not shown). Thus, the results regarding the proportion of studies indicating CENPM upregulation (Fig. 1A and B), the mRNA and protein expression levels of CENPM (Figs. 1C and D, and 2A) in tumor vs. normal samples, and the association between CENPM mRNA expression and tumor stage (Fig. 2B) and patient survival time (Fig. 2C) demonstrated that CENPM was highly associated with $\mathrm{BC}$ among various types of cancer and suggested that CENPM could be used as a prognostic marker for BC.

CENPM modulates a biological network including nine genes associated with the cell cycle in BC. Given the predictive 
A

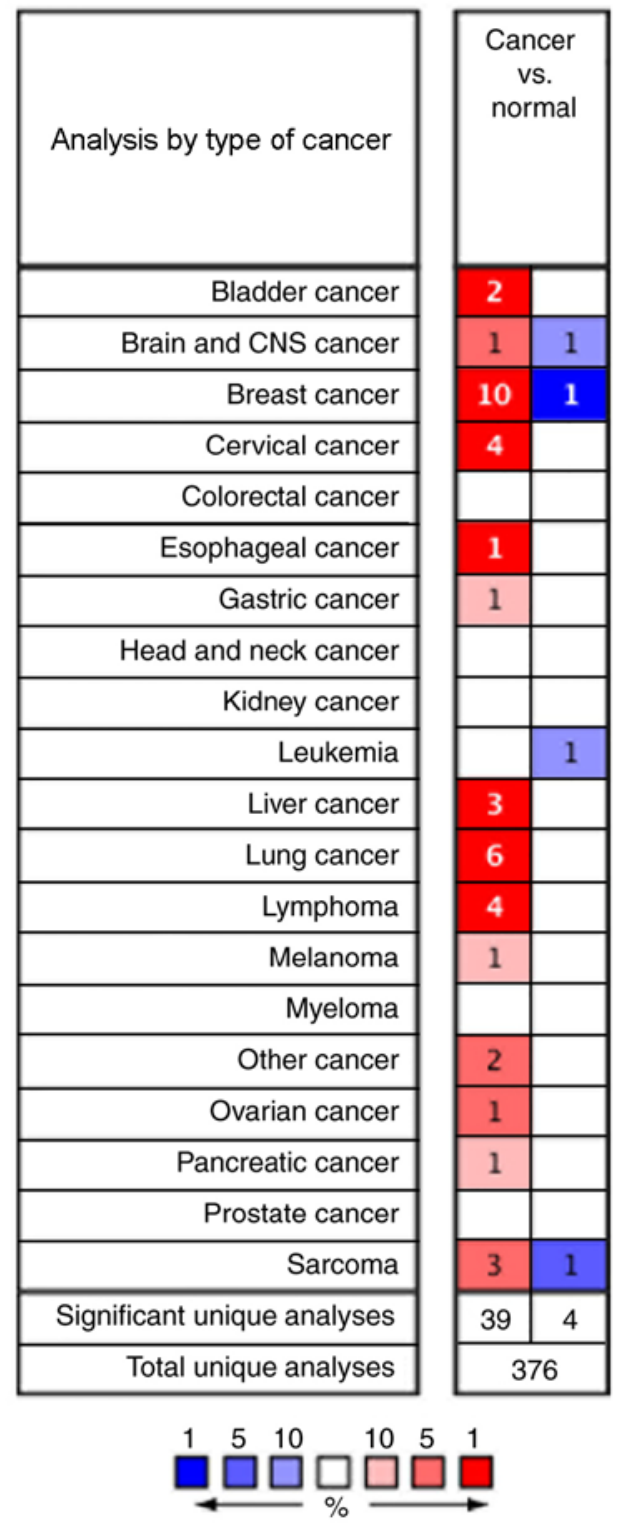

$\mathrm{B}$

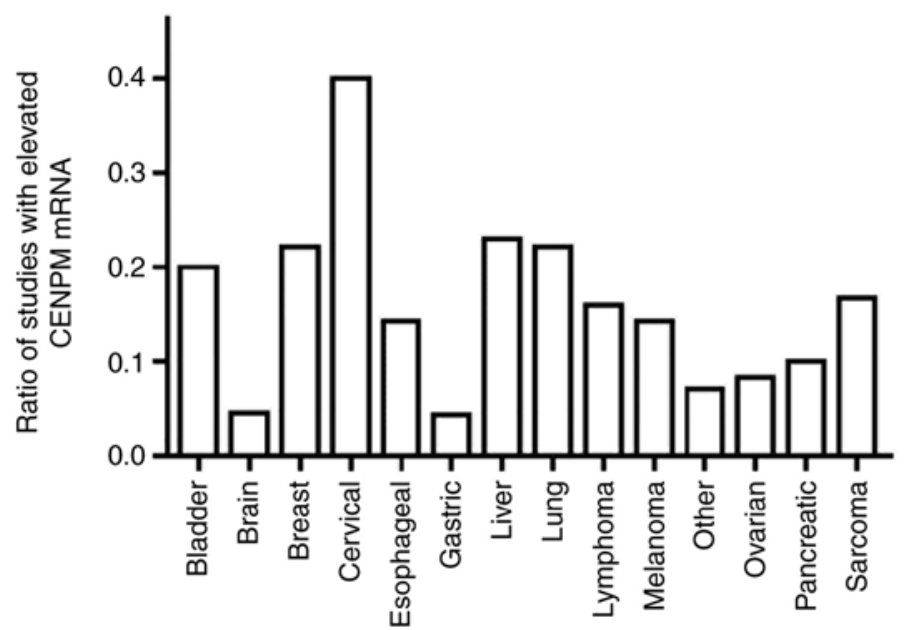

C

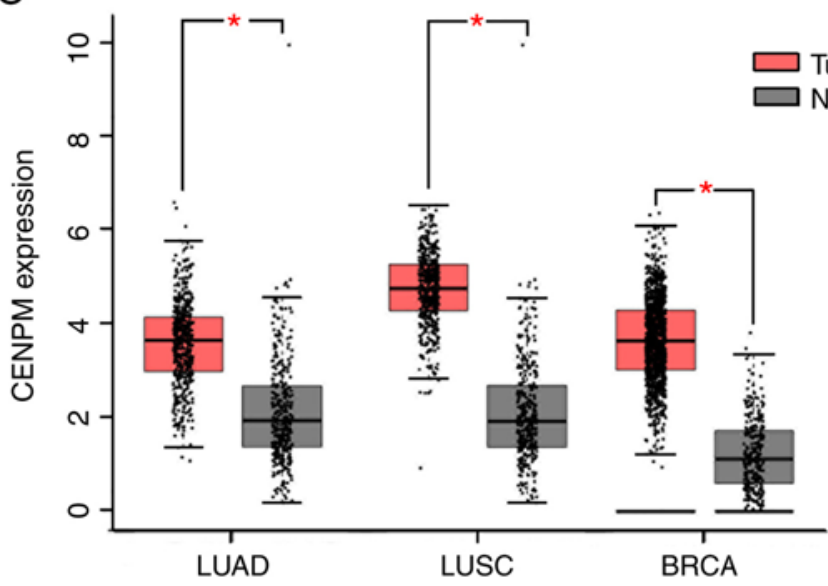

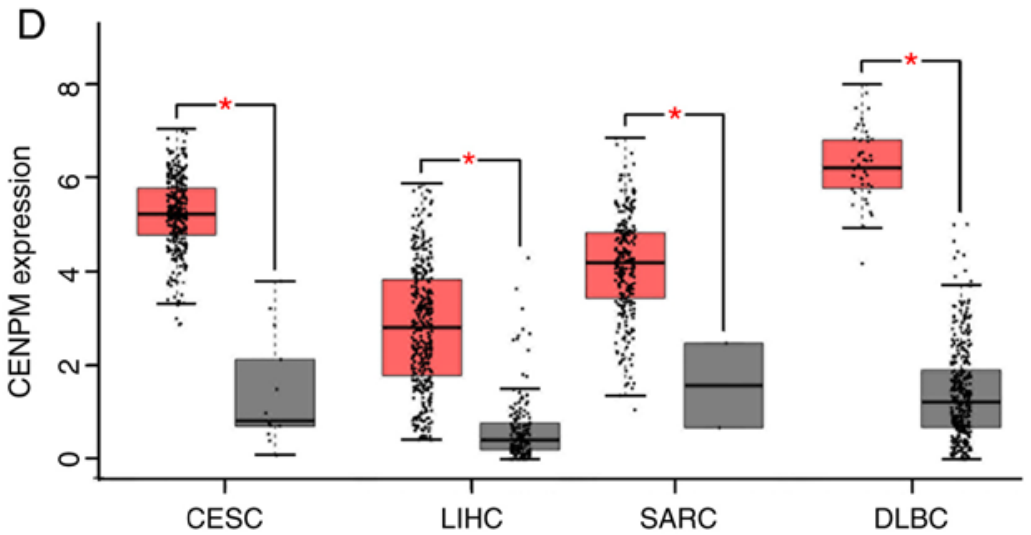

Figure 1. Expression levels of CENPM in various types of human cancer. (A) Analyses performed on data for various cancer types from the Oncomine database revealed that CENPM mRNA expression was increased in tumor samples compared with normal samples (fold change $>2$, $\mathrm{P}<1 \times 10^{-4}$, gene rank: Top 10\%). (B) Number of studies revealing CENPM mRNA upregulation relative to the total number of studies examined. The data were collected from the Oncomine database and included data for various cancer types (fold change $>2, \mathrm{P}<1 \times 10^{-4}$, gene rank: Top $10 \%$ ). (C and D) mRNA expression levels of CENPM were significantly higher in patients with LUAD $(n=483)$ vs. normal individuals $(n=347)$, patients with LUSC $(n=486)$ vs. normal individuals $(n=338)$, patients with BRCA ( $\mathrm{n}=1085)$ vs. normal individuals $(\mathrm{n}=291)$, patients with CESC $(\mathrm{n}=306)$ vs. normal individuals $(\mathrm{n}=13)$, patients with LIHC ( $\mathrm{n}=369)$ vs. normal individuals $(n=160)$, patients with SARC $(n=262)$ vs. normal individuals $(n=2)$ and patients with DLBC $(n=47)$ vs. normal individuals $(n=337)$ in Gene Expression Profiling Interactive Analysis. " $\mathrm{P}<0.05$. CENPM, centromere protein M; LUAD, lung adenocarcinoma; LUSC, lung squamous cell carcinoma; CESC, cervical squamous cell carcinoma and endocervical adenocarcinoma; LIHC, liver hepatocellular carcinoma; DLBC, diffuse large B-cell lymphoma; BRCA, invasive breast carcinoma; SARC, sarcoma.

function of CENPM, the hypothesis that the molecule could also serve as a prognostic marker in different types of $\mathrm{BC}$ was further tested using the Kaplan-Meier Plotter database (27). As shown in Fig. 3A, higher CENPM mRNA expression levels in patients with luminal A-type BC (339 patients, $\mathrm{P}=0.0052$ ) were highly associated with lower survival rates.
However, the expression levels of CENPM did not affect survival rate in luminal B-type (Fig. 3B; 77 patients, $\mathrm{P}=0.18$ ), HER2-positive-type (Fig. 3C; 115 patients, $\mathrm{P}=0.33$ ) or basal-like-type BC (Fig. 3D; 107 patients, $\mathrm{P}=0.71$ ).

To accurately determine the biological role of CENPM, 1,199 genes determined to be associated with CENPM by 
A

है
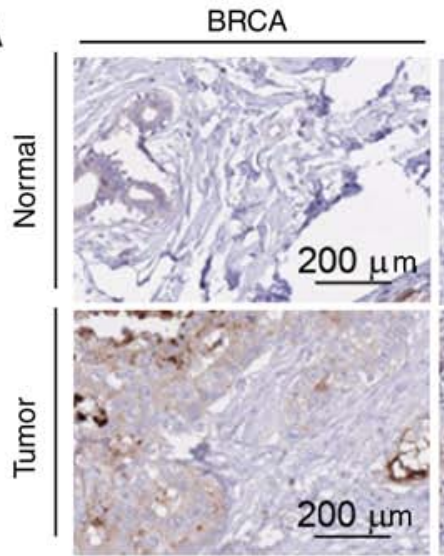

B

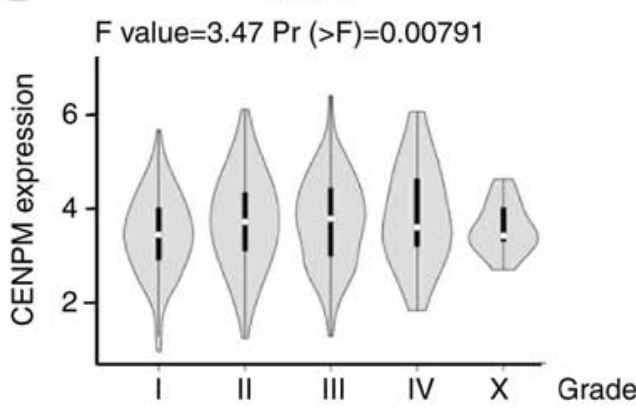

BRCA
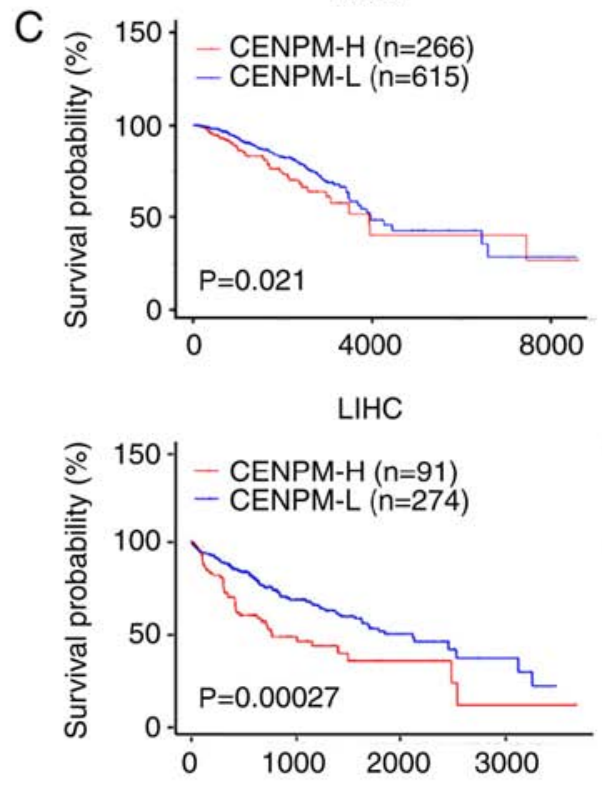

CESC

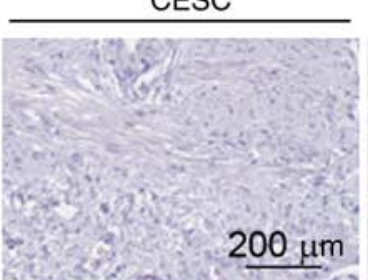

$200 \mu \mathrm{m}$
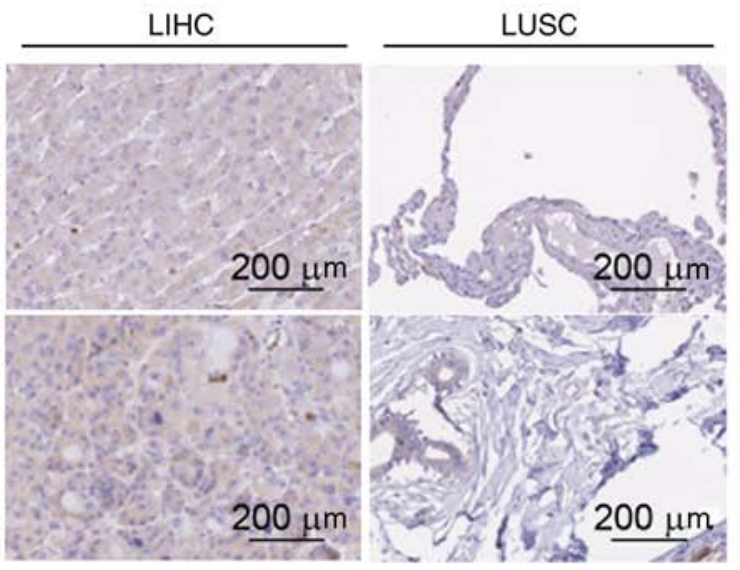

$200 \mu \mathrm{m}$

LIHC
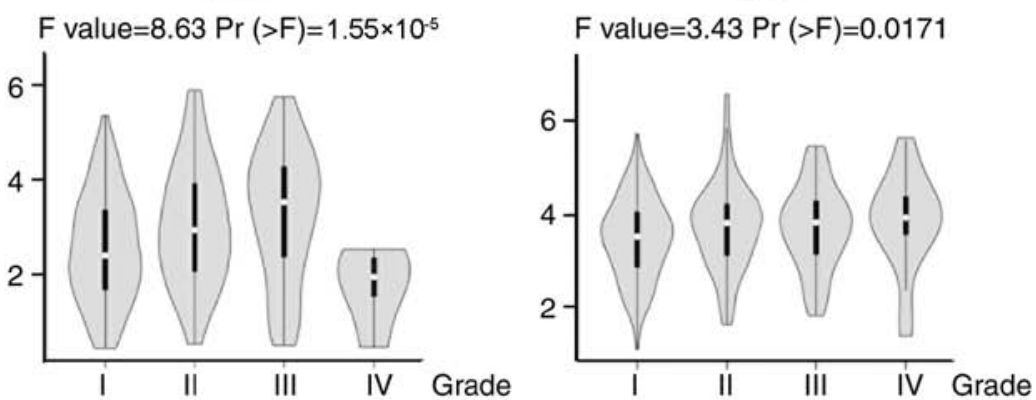

CESC
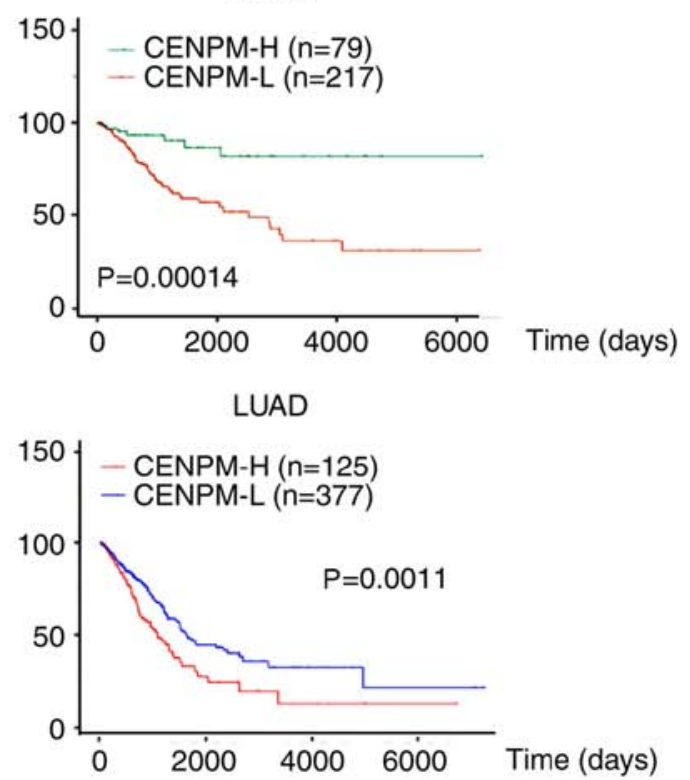

Figure 2. Expression levels of CENPM in various types of human cancer. (A) Immunohistochemical staining of CENPM protein levels in breast ( $\mathrm{n}=2 \mathrm{for}$ normal breast tissue and $n=2$ for BRCA tissue), cervix ( $n=2$ for normal cervical tissue and $n=2$ for CESC tissue), liver ( $n=3$ for normal liver tissue and $n=1$ for LIHC tissue) and lung ( $\mathrm{n}=2$ for normal lung tissue, and $\mathrm{n}=4$ for LUSC tissue) samples. The data for the samples, which were collected and stained with a CENPM antibody, were obtained from the Human Protein Atlas. Scale bars, $200 \mu \mathrm{m}$. (B) Data on CENPM mRNA expression levels in BRCA, LIHC, and LUAD of different stages were downloaded from the Gene Expression Profiling Interactive Analysis database. (C) Kaplan-Meier plotter analysis of 881 patients with BRCA, 296 patients with CESC, 365 patients with LIHC and 502 patients with LUAD who had high or low CENPM mRNA expression. CENPM, centromere protein M; BRCA, invasive breast carcinoma; CESC, cervical squamous cell carcinoma and endocervical adenocarcinoma; LIHC, liver hepatocellular carcinoma; LUSC, lung squamous cell carcinoma; LUAD, lung adenocarcinoma.

Spearman's rank correlation coefficient analysis (Spearman $>0.4$, $\mathrm{P}<0.001$ ) of 815 invasive breast carcinomas and one normal specimen were selected from the cBioPortal database (19). A circular correlation network (data not shown) with these genes was drawn that included 399 nodes and 11,495 edges according to the STRING database. Genes with degrees of connectivity (as calculated with CytoHubba in Cytoscape) $>100$ were selected and it was identified that the mRNA expression levels of RAD51 (Fig. 4A), KIF4A (Fig. 4B), RACGAP1 (Fig. 4C), SPAG5 (Fig. 4D), HMMR (Fig. 4E), CDC25C (Fig. 4F), CKS2 (Fig. 4G), ERCC6L 

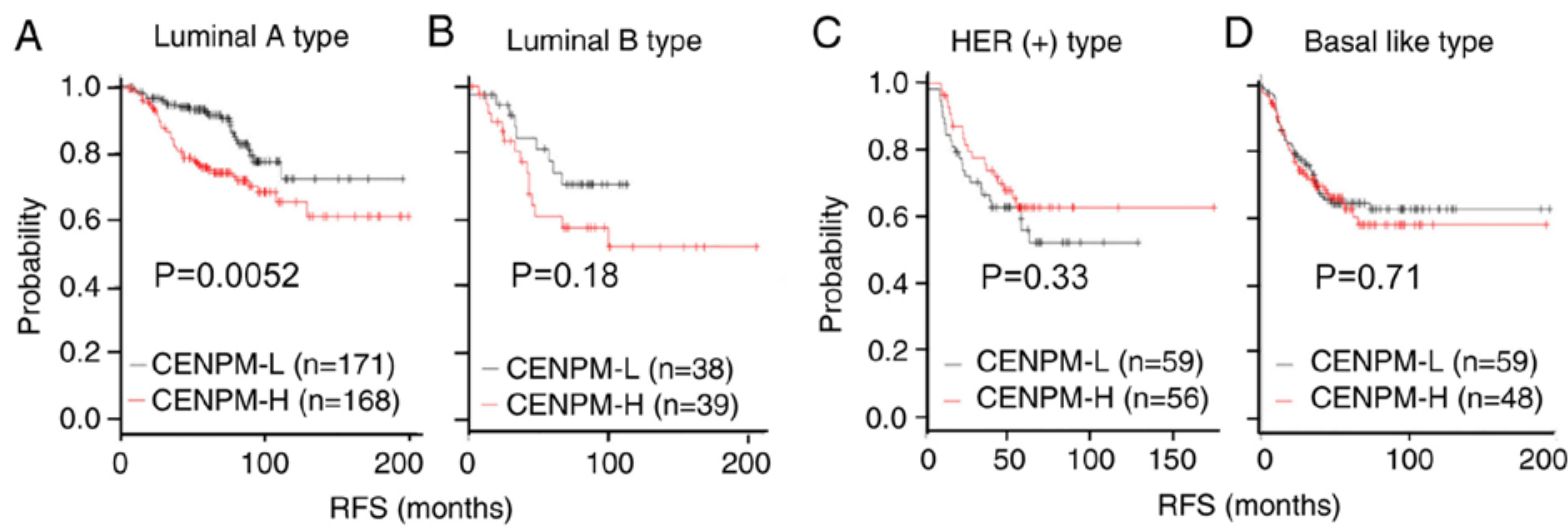

Figure 3. CENPM mRNA expression is associated with patient survival rate in breast cancer. Kaplan-Meier plotter analysis of (A) 339 patients with ER-positive, PR-positive and HER2-negative luminal A-type; (B) 77 patients with ER-positive, PR-negative and HER2-negative luminal B-type; (C) 115 patients with HER positive-type; and (D) 107 patients with basal-like-type breast cancer, who had high or low CENPM mRNA expression. CENPM, centromere protein M; ER, estrogen receptor; PR, progesterone receptor; HER2, human epidermal growth factor receptor 2; -L, low; -H, high; RFS, regression-free survival.
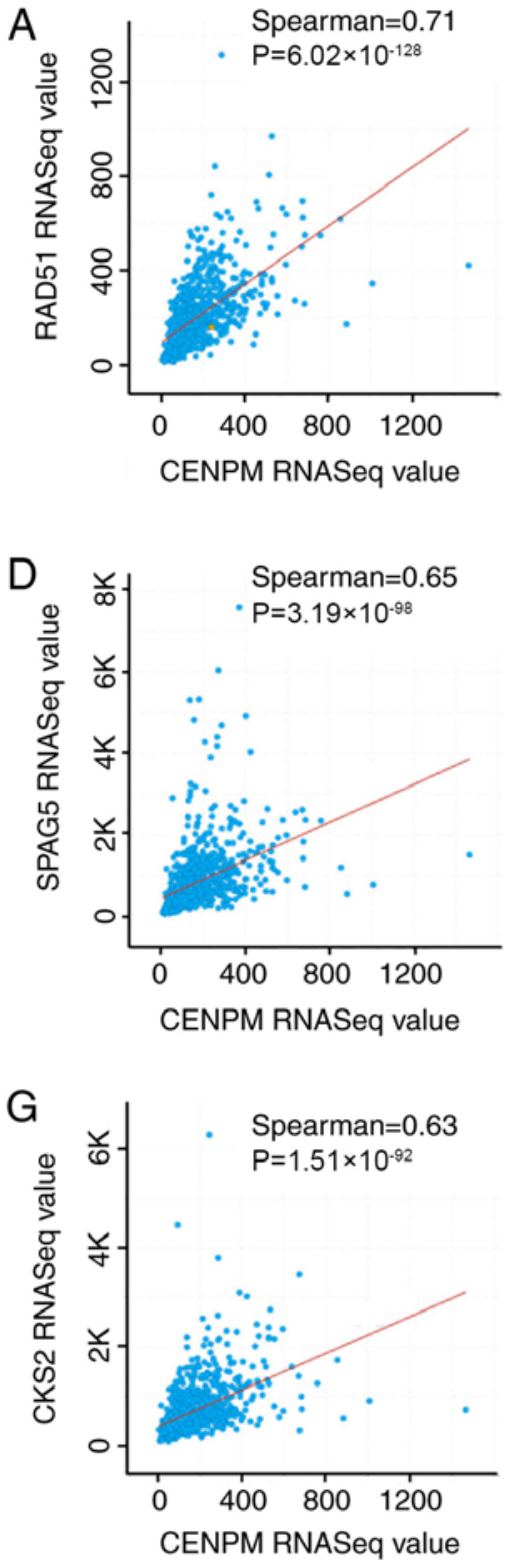
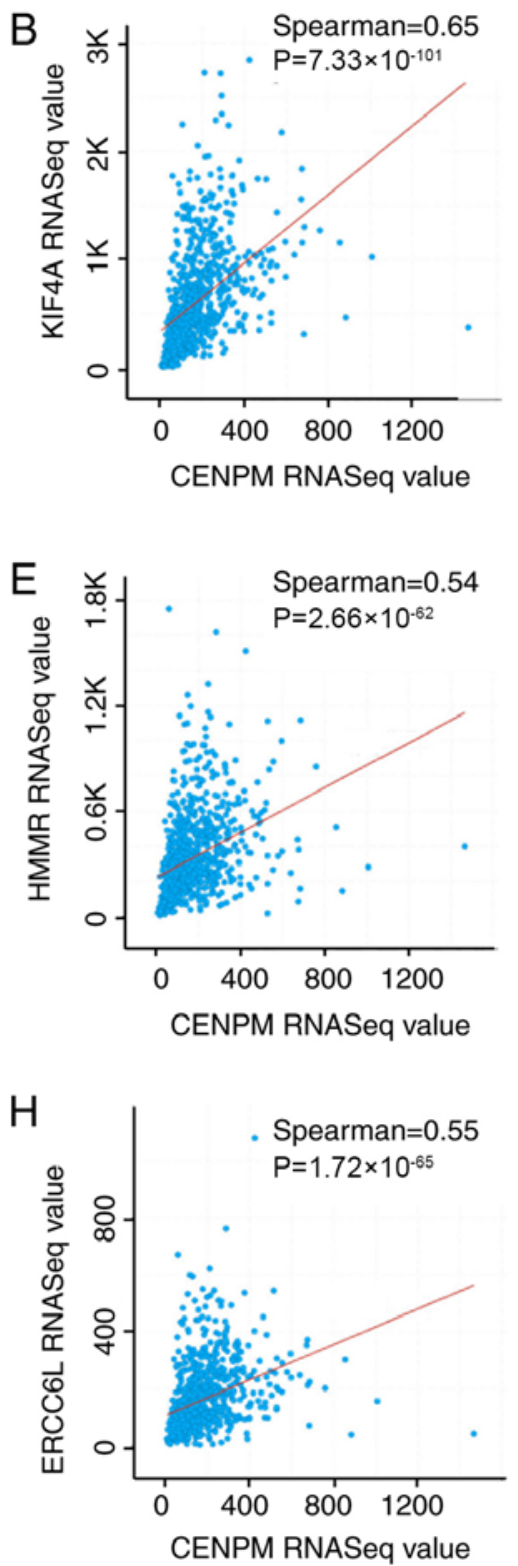
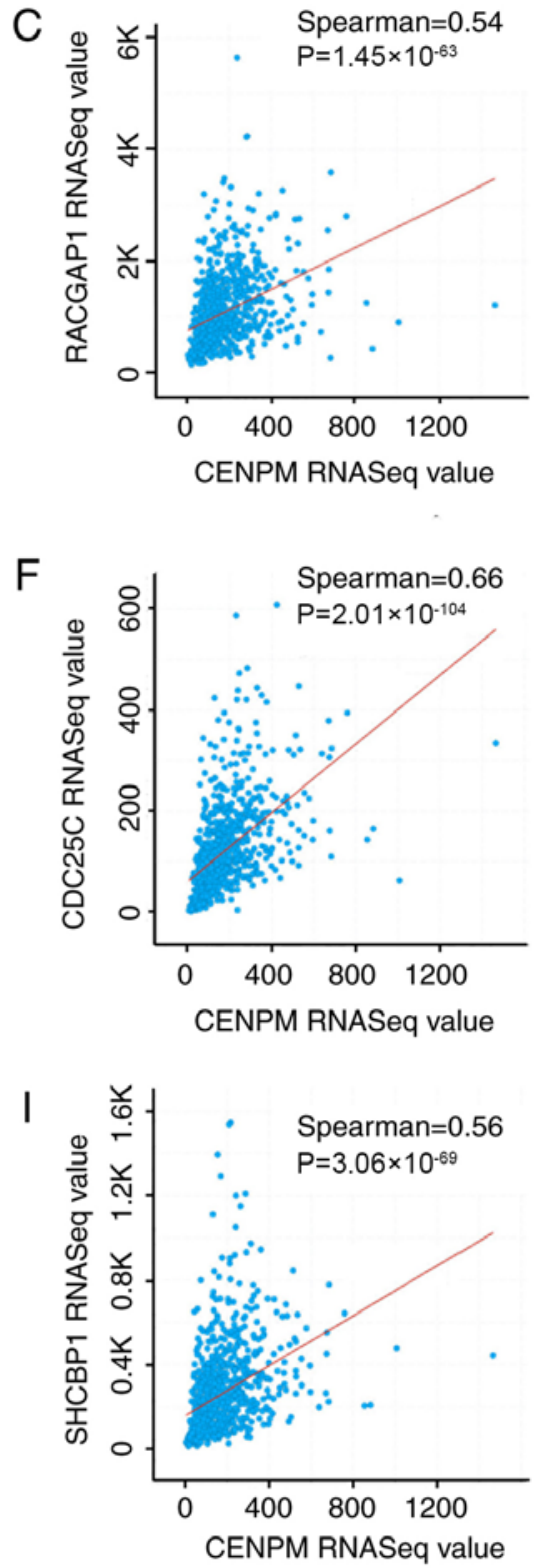

Figure 4. CENPM modulates a biological network including nine genes associated with the cell cycle in breast cancer. Spearman's correlation analyses were performed to analyze the association of CENPM mRNA expression with (A) RAD51, (B) KIF4A, (C) RACGAP1, (D) SPAG5, (E) HMMR, (F) CDC25C, (G) CKS2, (H) ERCC6L and (I) SHCBP1 mRNA expression. The data were downloaded from the cBioPortal database. CENPM, centromere protein M. 

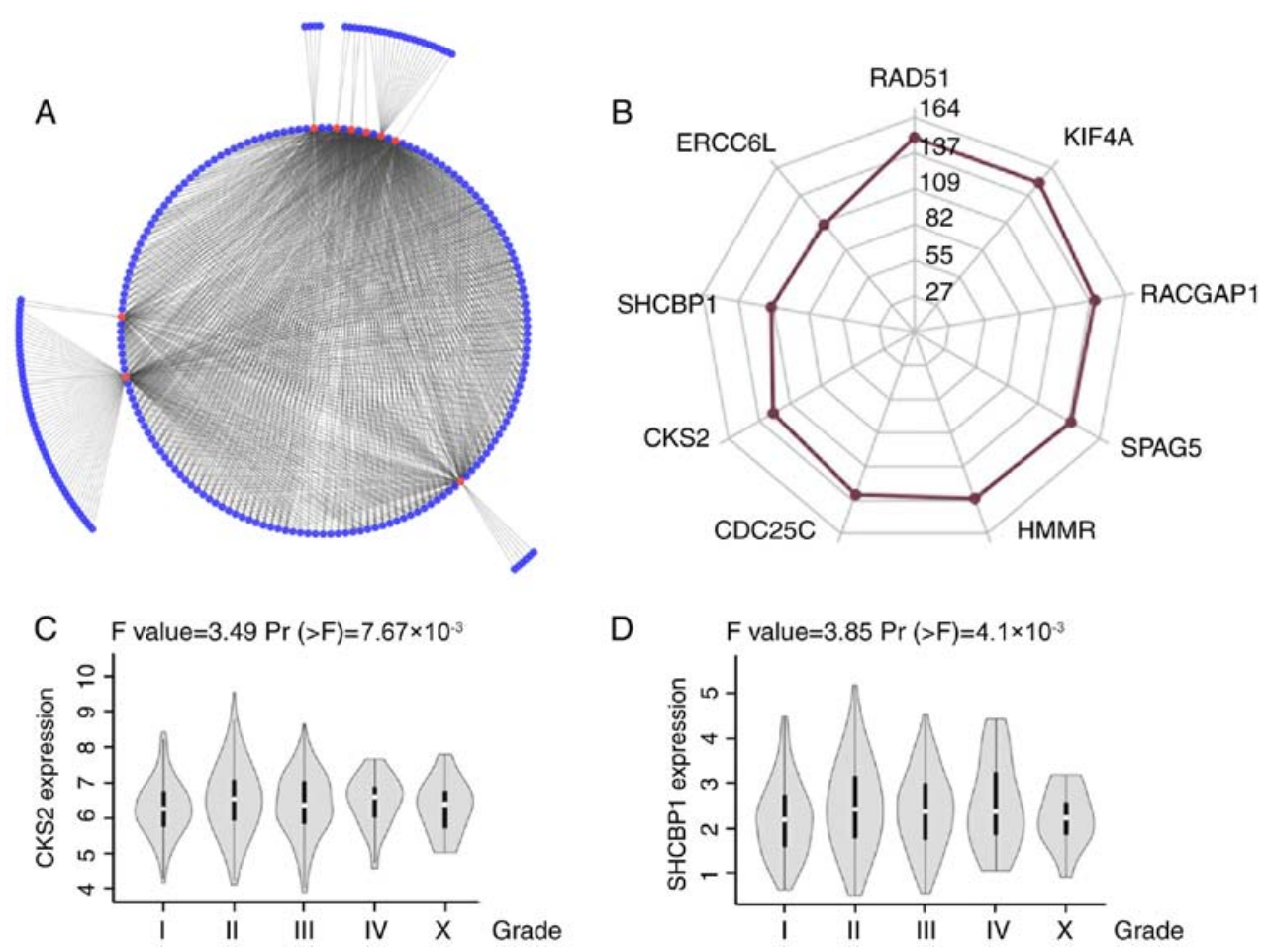

Figure 5. CENPM modulates a biological network including nine genes associated with the cell cycle in breast cancer. Genes determined to be associated with CENPM by Spearman's rank correlation coefficient analysis (Spearman $>0.4, \mathrm{P}<0.001$ ) of data from the cBioPortal database were selected to (A) draw a network, and (B) nine genes strongly correlated with CENPM were included in the network on the basis of their degrees of connectivity. Data on (C) CKS2 and (D) SHCBP1 mRNA expression in BRCA at different stages were downloaded from the Gene Expression Profiling Interactive Analysis database. CENPM, centromere protein M; BRCA, invasive breast carcinoma.

(Fig. 4H) and SHCBP1 (Fig. 4I) were positively correlated with those of CENPM in BRCA. In addition, these nine genes were connected by 207 of 399 nodes (Fig. 5A and B), suggesting the critical functions of these nine genes in the correlation network. Consistent with these findings, RT-qPCR analysis revealed that ERCC6L, CKS2, KIF4A, SPAG5 and CDC25C mRNA expression levels were significantly increased in MCF7 cells transfected with a CENPM overexpression vector (Fig. 6A); the protein expression levels of CKS2 were also identified to be increased in MCF7 cells with elevated CENPM expression (Fig. S1). However, the mRNA expression levels of SHCBP1, RAD51, HMMR and RACGAP1 were only slightly and not significantly upregulated in CENPM-overexpressing MCF7 cells ( $\mathrm{P}>0.05$; Fig. 6A).

The results of biological experiments and TCGA database analyses confirmed that these nine genes were commonly upregulated in $\mathrm{BC}$ vs. normal samples; in addition, they were associated with tumor grade or mortality and involved in DNA replication, DNA repair or chromosome segregation (Table II). Next, a positive association was demonstrated between CKS2/SHCBP1 mRNA expression and tumor grade, which, to the best of our knowledge, has not previously been reported (Fig. 5C and D). These findings indicated that CENPM could serve as a prognostic marker in luminal A-type $\mathrm{BC}$ and may regulate tumorigenesis by altering cell cycle-associated proteins.

Upregulation of CENPM enhances tumorigenesis in vitro. To study the precise effects of CENPM on BC, a CENPM overexpression plasmid was constructed and CENPM mRNA expression levels were detected in MCF7 cells transfected with a control vector vs. the CENPM overexpression vector (Fig. 6A). MCF7 cells (Fig. 6B) and T-47D cells (Fig. S2) transfected with the CENPM overexpression vector proliferated more quickly than the normal control cells, as determined by CCK-8 assay. Conversely, MCF7 cells transfected with CENPM shRNA proliferated more slowly than control cells (Fig. S3A and B). Next investigated were the proliferation-associated gene markers, including Ki67 and proliferating cell nuclear antigen (PCNA), with RT-qPCR assays, which demonstrated that the mRNA expression levels of Ki67 and PCNA were elevated in CENPM-overexpressing MCF7 cells (Fig. 6C). Considering that the expression of numerous cell cycle-related genes was identified to be positively associated with CENPM expression, it was hypothesized that CENPM may enhance proliferation by affecting cells in mitosis. The fluorescence-activated cell sorting results (Fig. 6D) demonstrated that the proportion of MCF7 cells in $S$ phase was elevated and that the proportion of cells in $\mathrm{G}_{1}$ phase was decreased following transfection with the CENPM overexpression vector, supporting the hypothesis that CENPM accelerated the progression of cells through $\mathrm{G}_{1}$ phase to promote tumor growth.

Since tumorigenesis and migratory ability are key factors associated with tumor malignancy (28), a colony formation assay was conducted to assess the tumorigenicity of CENPM. As shown in Fig. 6E and 6F, CENPM-overexpressing MCF7 cells formed significantly more colonies compared with control vector-expressing MCF7 cells. However, the wound-healing assay demonstrated that CENPM had little 

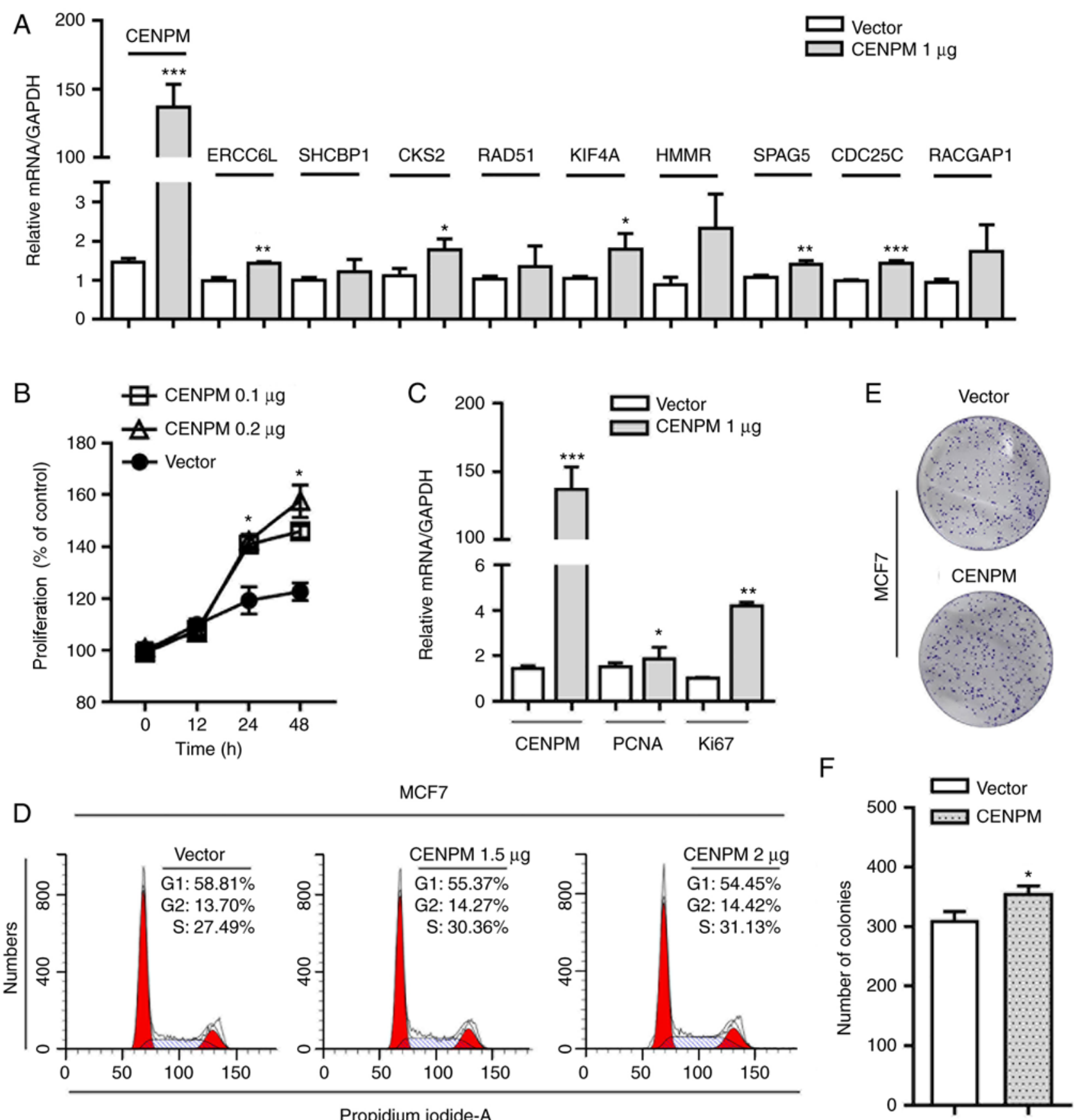

Figure 6. Upregulation of CENPM enhances tumorigenesis in vitro. (A) Following transfection of MCF7 cells with a CENPM-overexpression vector or a control vector for $24 \mathrm{~h}$, the mRNA expression levels of nine CENPM-related genes were detected. (B) MCF7 cells in 96-well plates were transfected with an empty vector or with 0.1 or $0.2 \mu \mathrm{g}$ CENPM plasmid per well for $12 \mathrm{~h}$, and the proliferation of cells was detected by CCK-8 assay after another 12,24 or 48 h. ${ }^{*} \mathrm{P}<0.05 . \mathrm{n}=6$. (C) mRNA expression levels of CENPM, PCNA and Ki67 were detected by reverse transcription-quantitative PCR. ${ }^{*} \mathrm{P}<0.05$, ${ }^{* *} \mathrm{P}<0.01$, ${ }^{*}{ }^{* *} \mathrm{P}<0.001$ vs. the corresponding control group $(\mathrm{n}=3)$. (D) Cell cycle distribution was analyzed by fluorescence-activated cell sorting. (E and $\mathrm{F}$ ) Vector or CENPM-overexpressing MCF7 cells were cultured in 6-well plates with medium containing 5\% FBS for 1 week, and then the cells were stained with crystal violet and imaged. CENPM, centromere protein $\mathrm{M}$.

effect on the migration of MCF7 cells; and migration rate was $20 \%$ in both groups (Fig. S4).

As imbalance between cell division and apoptosis leads to tumorigenesis, it was hypothesized that investigation of the role of CENPM in apoptosis could further elucidate the precise function of CENPM in BC. To test this hypothesis, a TUNEL assay was conducted and the results revealed that the amount of DNA damage was decreased in CENPM-overexpressing MCF7 cells (Fig. 7A and B). In addition, western blot analysis with anti-Bcl-2, anti-Bax and anti-cleaved caspase-3 antibodies further demonstrated the inhibitory role of CENPM in apoptosis (Fig. 7C and D). Therefore, overexpression of CENPM in MCF7 cells accelerated the development of a BC-like state by interfering with proliferation and apoptosis in vitro.

CENPM gene mutations. The COSMIC database (v89), was used to evaluate whether the CENPM gene was substantially mutated among various types of human cancer. The expression 
Table II. The nine critical genes significantly connected with centromere protein $\mathrm{M}$.

\begin{tabular}{|c|c|c|c|}
\hline Gene & Degree & $\begin{array}{l}\text { Kaplan-meier survival } \\
\text { P-value in BRCA }\end{array}$ & Function \\
\hline RAD51 & 149 & 0.0045 & Participates in homologous strand exchange, an important process in DNA repair \\
\hline KIF4A & 149 & 0.036 & $\begin{array}{l}\text { Translocates transcription factor pcr } 1 \text { to the plus ends of interdigitating spindle } \\
\text { microtubules during the metaphase to anaphase transition }\end{array}$ \\
\hline RACGAP1 & 140 & 0.00095 & $\begin{array}{l}\text { Controls cytokinesis by forming the central spindling complex and mediates the } \\
\text { rho-dependent signaling required for actomyosin contractile ring assembly }\end{array}$ \\
\hline SPAG5 & 138 & 0.019 & $\begin{array}{l}\text { Essential component of the mitotic spindle required for normal chromosome } \\
\text { segregation and progression into anaphase }\end{array}$ \\
\hline HMMR & 135 & 0.027 & $\begin{array}{l}\text { Forms a complex with BRCA, which is a key gene associated with } \mathrm{G} 2 \text { to } \mathrm{M} \text { transition } \\
\text { in breast cancer }\end{array}$ \\
\hline $\mathrm{CDC} 25 \mathrm{C}$ & 132 & 0.03 & Triggers mitosis in the cell cycle \\
\hline CKS2 & 124 & 0.012 & Promotes cell cycle progression by triggering degradation of p27 \\
\hline SHCBP1 & 111 & 0.031 & Serves a role in signaling pathways to govern cellular proliferation \\
\hline ERCC6L & 107 & 0.00071 & Acts as an essential component of the spindle assembly checkpoint \\
\hline
\end{tabular}

BRCA, invasive breast carcinoma.
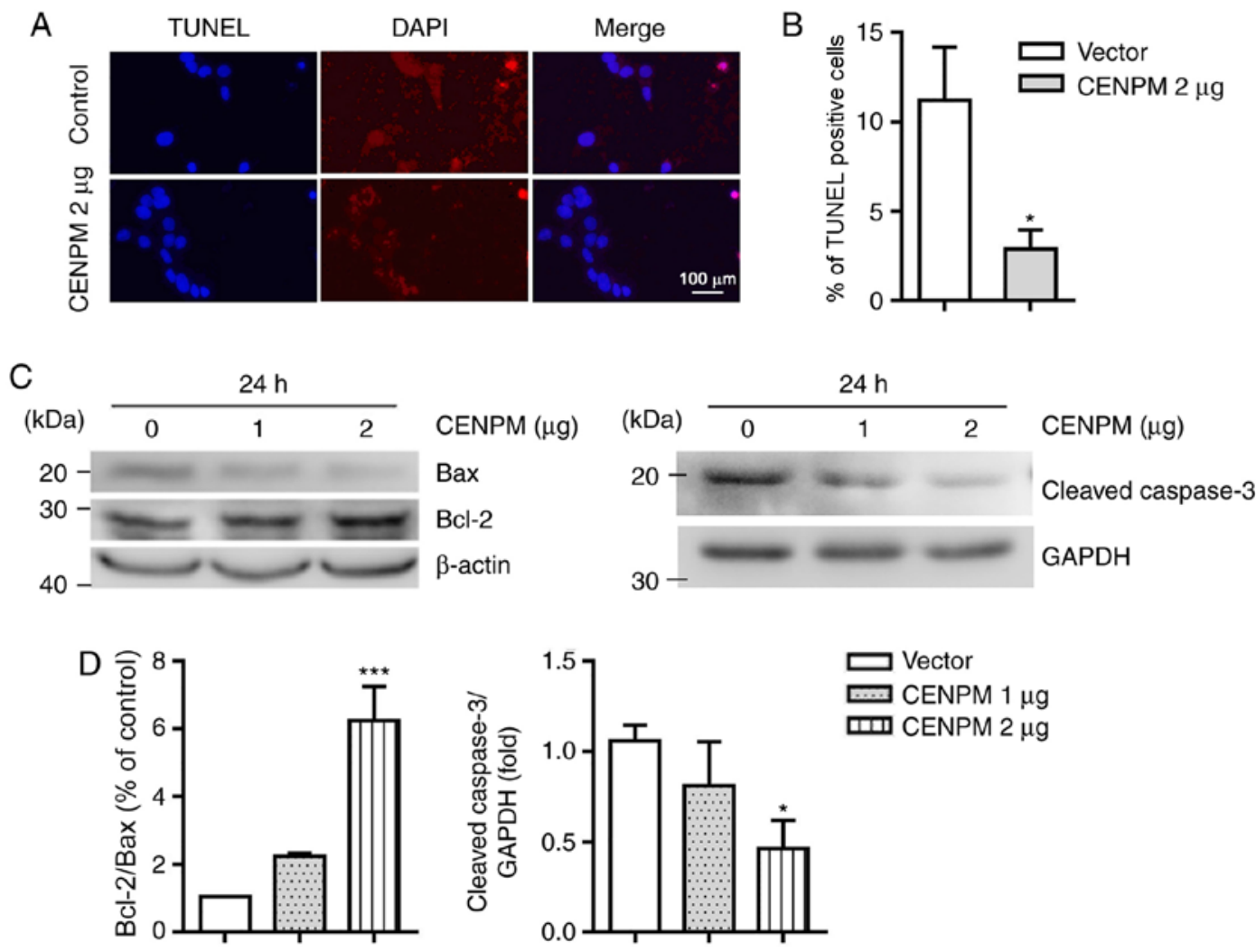

Figure 7. Upregulation of CENPM enhances tumorigenesis in vitro. (A and B) Apoptosis was measured with a one-step TUNEL apoptosis assay kit, and $(\mathrm{C}$ and $\mathrm{D})$ apoptosis-related protein levels were measured by western blot analysis with anti-Bcl-2, anti-Bax, and anti-cleaved caspase-3 antibodies. ${ }^{*} \mathrm{P}<0.05$. ${ }^{* * * *} \mathrm{P}<0.001$. n.s, not significant. $\mathrm{n}=3$. CENPM, centromere protein $\mathrm{M}$.

of CENPM was examined in 47,818 patient tissues representing 39 distinct types of cancer from inception to May 15, 2019. Of the 39 cancer types, 19 were associated with CENPM gene mutations, primarily 37 point mutations (total percentage of mutated, $0.077 \%$ ), and two frameshift deletions (total percentage of mutated, $0.004 \%$ ), and the corresponding specimens were further examined. No insertions or complex mutations were identified in the samples (Table III). Among the CENPM gene point mutations, 19 were missense substitutions, and 18 were synonymous substitutions with no changes in the protein-coding sequence. Point mutations occurred in the skin (gene mutation rate, $0.54 \%$ ), large intestine (gene mutation rate, $0.39 \%$ ) and 
Table III. Centromere protein M gene mutations among 47,818 patients with cancer (Catalogue of Somatic Mutations in Cancer database).

\begin{tabular}{lcc}
\hline Mutation type & Number & Ratio (\%) \\
\hline Nonsense substitution & 0 & 0 \\
Missense substitution & 19 & 0.039 \\
Synonymous substitution & 18 & 0.038 \\
Inframe insertion & 0 & 0 \\
Frameshift insertion & 0 & 0 \\
Inframe deletion & 0 & 0 \\
Frameshift deletion & 2 & 0.004 \\
Complex mutation & 0 & 0 \\
\hline
\end{tabular}

endometrium (gene mutation rate, $0.42 \%$ ). Overall, considering the low gene mutation rates, CENPM gene sequence changes may not be the main reasons for tumorigenesis.

\section{Discussion}

CENPM gene expression has been reported to be associated with tumorigenesis in primary melanoma, bladder cancer, hepatocellular carcinoma, and head and neck squamous cell carcinoma. The present study analyzed the association of 13 types of cancer (bladder, brain, breast, cervical, esophageal, gastric, liver, lung, ovarian and pancreatic cancer, and lymphoma, melanoma and sarcoma) with ectopic CENPM mRNA expression, and identified that CENPM protein expression was upregulated in patients with BRCA and CESC. In addition, it was revealed that CENPM mRNA expression levels were positively associated with tumor grade in BRCA, LIHC and LUAD, and with mortality in BRCA, CESC, LIHC and LUAD. Based on the BC cell types of origin, the critical role of CENPM was further confirmed in luminal A-type BC mortality. In general, the present study implied that exploration of CENPM expression may enhance the diagnostic accuracy and survival probability in patients with cancer, particularly in patients with BC.

The protein interaction network identified nine key genes [RAD51 (29), KIF4A (30), RACGAP1 (31), SPAG5 (32), HMMR (33), CDC25C (34), CKS2 (35), SHCBP1 (36) and ERCC6L (37)] that interacted strongly with CENPM mRNA. Subsequently, the functions of these nine genes (38-46) were profiled in various types of cancer and it was identified that all of them have been reported to accelerate the progression of cancer, including BC and hepatocellular carcinoma (29-37). RT-qPCR demonstrated that the expression levels of five of the nine genes, ERCC6L, CKS2, KIF4A, SPAG5 and CDC25C, were increased in MCF7 cells with elevated CENPM expression, implying that CENPM may promote tumor progression by controlling the expression of these five genes. Notably, normal cells utilize $\mathrm{CDC} 25 \mathrm{C}$ to remove the inhibitory phosphates and trigger the $\mathrm{G}_{2}$-prophase transition (47), and utilize SPAG5 (32), KIF4A (48), ERCC6L (49) or CKS2 (50) to properly position chromosomes during metaphase. Of the nine genes, four (SHCBP1, RAD51, HMMR and RACGAP1), were identified as being associated with CENPM through cBioPortal correlation analysis; however, the mRNA expression levels of these genes remained unchanged in CENPM-overexpressing MCF7 cells. Further exploration is required to elucidate the mechanism by which CENPM can regulate the expression of ERCC6L, CKS2, KIF4A, SPAG5 and $\mathrm{CDC} 25 \mathrm{C}$ in mitosis, and to confirm whether the CENPM protein interacts with the SHCBP1, RAD51, HMMR and RACGAP1 proteins. In addition, the present study demonstrated that CENPM-overexpression promoted cell cycle progression in human BC cells, and decreased the apoptotic rate, as calculated by TUNEL assay. Taken together, these results indicated that ectopic CENPM expression may accelerate the development of a BC-like state and control mitosis by regulating the expression of KIF4A, RECC6L, CKS2, CDC25C and SPAG5, and affect apoptosis in BC cells.

In order to explain why CENPM expression was upregulated in patients with BRCA, point mutations, insertions, deletions or complex mutations of CENPM were identified in the present study; calculating the CENPM gene mutation rates among various types of cancer may be helpful for revealing the precise function of CENPM. Based on the low CENPM mutation rates, the present study suggested that CENPM gene sequence changes (point mutations) may not be the main reason underlying its effects on tumorigenesis. Although mutations in proto-oncogenes or tumor suppressor genes may be major drivers of carcinogenesis, the epigenetic modification (including DNA methylation, acetylation, phosphorylation and ubiquitylation) of CENPM may still serve a critical role in gene expression regulation, which is also considered an important factor for tumorigenesis (51). Thus, it is suggested that further experiments should be conducted to explain which pathway contributes to the upregulation of CENPM in BC cells.

In conclusion, the findings of the present study indicated that upregulation of CENPM may enhance $\mathrm{BC}$ progression via numerous mechanisms, including increasing upregulation of genes linked to cell proliferation and reducing cell apoptosis. The present study revealed, for the first time to the best of our knowledge, that CENPM may be a novel therapeutic target for $\mathrm{BC}$ progression.

\section{Acknowledgements}

Not applicable.

\section{Funding}

The present study was supported by the National Natural Science Foundation of China (grant no. 81960655) to Y Liu, and the Guizhou Medical University start-up fund for doctoral talent [grant no. J-(2018)014].

\section{Availability of data and materials}

The datasets used during the present study are available from the corresponding author upon reasonable request.

\section{Authors' contributions}

YL and WY performed the experiments; YL wrote and revised the manuscript; PR and TZ analyzed the data. All authors read and approved the final manuscript. 


\section{Ethics approval and consent to participate}

Not applicable.

\section{Patient consent for publication}

Not applicable.

\section{Competing interests}

The authors declare that they have no competing interests.

\section{References}

1. Smith RA, Andrews KS, Brooks D, Fedewa SA, Manassaram-Baptiste D, Saslow D and Wender RC: Cancer screening in the United States, 2019: A review of current American cancer society guidelines and current issues in cancer screening. CA Cancer J Clin 69: 184-210, 2019.

2. Vuong D, Simpson PT, Green B, Cummings MC and Lakhani SR: Molecular classification of breast cancer. Virchows Arch 465 1-14, 2014.

3. Li G, Cottier M, Sabido O, Gentil-Perret A, Lambert C, Passebosc-Faure K, Genin C and Tostain J: The in vivo DNA aneuploidization during expansion of conventional renal cell carcinoma. In vivo 16: 341-344, 2002.

4. Wu Q, Li B, Liu L, Sun S and Sun S: Centrosome dysfunction: A link between senescence and tumor immunity. Signal Transduct Target Ther 5: 107, 2020.

5. Howman EV, Fowler KJ, Newson AJ, Redward S, MacDonald AC, Kalitsis P and Choo KH: Early disruption of centromeric chromatin organization in centromere protein A (Cenpa) null mice. Proc Natl Acad Sci USA 97: 1148-1153, 2000.

6. Meluh PB and Koshland D: Evidence that the MIF2 gene of saccharomyces cerevisiae encodes a centromere protein with homology to the mammalian centromere protein CENP-C. Mol Biol Cell 6: 793-807, 1995.

7. Liu ST, Hittle JC, Jablonski SA, Campbell MS, Yoda K and Yen TJ: Human CENP-I specifies localization of CENP-F, MAD1 and MAD2 to kinetochores and is essential for mitosis. Nat Cell Biol 5: 341-345, 2003.

8. Fukagawa T, Mikami Y, Nishihashi A, Regnier V, Haraguchi T, Hiraoka Y, Sugata N, Todokoro K, Brown W and Ikemura T: CENP-H, a constitutive centromere component, is required for centromere targeting of CENP-C in vertebrate cells. EMBO J 20: 4603-4617, 2001

9. BasilicoF,Maffini S, WeirJR,PrumbaumD, Rojas AM,Zimniak T, De Antoni A, Jeganathan S, Voss B, van Gerwen S, et al: The pseudo GTPase CENP-M drives human kinetochore assembly. eLife 3: e02978, 2014.

10. Foltz DR, Jansen LE, Black BE, Bailey AO, Yates JR III and Cleveland DW: The human CENP-A centromeric nucleosomeassociated complex. Nat Cell Biol 8: 458-469, 2006.

11. Chen J, Wu F, Shi Y, Yang D, Xu M, Lai Y and Liu Y: Identification of key candidate genes involved in melanoma metastasis. Mol Med Rep 20: 903-914, 2019.

12. Kim WT, Seo SP, Byun YJ, Kang HW, Kim YJ, Lee SC, Jeong P, Song HJ, Choe SY, Kim DJ, et al: The anticancer effects of garlic extracts on bladder cancer compared to cisplatin: A common mechanism of action via centromere protein M. Am J Chin Med 46: 689-705, 2018.

13. Yu Z, Wang R, Chen F, Wang $J$ and Huang $X$ : Five novel oncogenic signatures could be utilized as AFP-related diagnostic biomarkers for hepatocellular carcinoma based on next-generation sequencing. Dig Dis Sci 63: 945-957, 2018.

14. Prystowsky MB, Adomako A, Smith RV, Kawachi N, McKimpson W, Atadja P, Chen Q, Schlecht NF, Parish JL, Childs $\mathrm{G}$ and Belbin TJ: The histone deacetylase inhibitor LBH589 inhibits expression of mitotic genes causing G2/M arrest and cell death in head and neck squamous cell carcinoma cell lines. J Pathol 218: 467-477, 2009.

15. Huang PJ, Chiu LY, Lee CC, Yeh YM, Huang KY, Chiu CH and Tang P: mSignatureDB: A database for deciphering mutational signatures in human cancers. Nucleic Acids Res 46(D1): D964-D970,2018.
16. Forbes SA, Beare D, Boutselakis H, Bamford S, Bindal N, Tate J, Cole CG, Ward S, Dawson E, Ponting L, et al: COSMIC: Somatic cancer genetics at high-resolution. Nucleic Acids Res 45(D1): D777-D783, 2017.

17. Rhodes DR, Kalyana-Sundaram S, Mahavisno V, Varambally $R$, Yu J, Briggs BB, Barrette TR, Anstet MJ, Kincead-Beal C, Kulkarni P, et al: Oncomine 3.0: Genes, pathways, and networks in a collection of 18,000 cancer gene expression profiles. Neoplasia 9: 166-180, 2007.

18. Tang Z, Li C, Kang B, Gao G, Li C and Zhang Z: GEPIA: A web server for cancer and normal gene expression profiling and interactive analyses. Nucleic Acids Res 45(W1): W98-W102, 2017.

19. Ciriello G, Gatza ML, Beck AH, Wilkerson MD, Rhie SK, Pastore A, Zhang H, McLellan M, Yau C, Kandoth C, et al: Comprehensive molecular portraits of invasive lobular breast cancer. Cell 163: 506-519, 2015.

20. Gao J, Aksoy BA, Dogrusoz U, Dresdner G, Gross B, Sumer SO, Sun Y, Jacobsen A, Sinha R, Larsson E, et al: Integrative analysis of complex cancer genomics and clinical profiles using the cBioPortal. Sci Signal 6: pl1, 2013.

21. von Mering C, Huynen M, Jaeggi D, Schmidt S, Bork P and Snel B: STRING: A database of predicted functional associations between proteins. Nucleic Acids Res 31: 258-261, 2003.

22. Chandrashekar DS, Bashel B, Balasubramanya SAH, Creighton CJ, Ponce-Rodriguez I, Chakravarthi B and Varambally S: UALCAN: A portal for facilitating tumor subgroup gene expression and survival analyses. Neoplasia 19: 649-658, 2017.

23. Hou GX, Liu P, Yang J and Wen S: Mining expression and prognosis of topoisomerase isoforms in non-small-cell lung cancer by using oncomine and kaplan-meier plotter. PLoS One 12: e $0174515,2017$.

24. Livak KJ and Schmittgen TD: Analysis of relative gene expression data using real-time quantitative PCR and the 2(-Delta Delta C(T)) method. Methods 25: 402-408, 2001.

25. Lackler KP, Cochran DL, Hoang AM, Takacs V and Oates TW: Development of an in vitro wound healing model for periodontal cells. J Periodontol 71: 226-237, 2000.

26. Moore CB, Guthrie EH, Huang MT and Taxman DJ: Short hairpin RNA (shRNA): Design, delivery, and assessment of gene knockdown. Methods Mol Biol 629: 141-158, 2010.

27. Gyorffy B, Lanczky A, Eklund AC, Denkert C, Budczies J, Li Q and Szallasi Z: An online survival analysis tool to rapidly assess the effect of 22,277 genes on breast cancer prognosis using microarray data of 1,809 patients. Breast Cancer Res Treat 123: 725-731, 2010.

28. Mierke CT: The matrix environmental and cell mechanical properties regulate cell migration and contribute to the invasive phenotype of cancer cells. Rep Prog Phys 82: 064602, 2019.

29. Ward A, Khanna KK and Wiegmans AP: Targeting homologous recombination, new pre-clinical and clinical therapeutic combinations inhibiting RAD51. Cancer Treat Rev 41: 35-45, 2015.

30. Hu G, Yan Z, Zhang C, Cheng M, Yan Y, Wang Y, Deng L, Lu Q and Luo S: FOXM1 promotes hepatocellular carcinoma progression by regulating KIF4A expression. J Exp Clin Cancer Res 38: 188,2019

31. Wang MY, Chen DP, Qi B, Li MY, Zhu YY, Yin WJ, He L, Yu Y, Li ZY, Lin L, et al: Pseudogene RACGAP1P activates RACGAP1/Rho/ERK signalling axis as a competing endogenous RNA to promote hepatocellular carcinoma early recurrence. Cell Death Dis 10: 426, 2019.

32. Li M, Li A, Zhou S, Lv H and Yang W: SPAG5 upregulation contributes to enhanced c-MYC transcriptional activity via interaction with c-MYC binding protein in triple-negative breast cancer. J Hematol Oncol 12: 14, 2019.

33. Cai Y, Sheng Z, Chen Y and Wang J: LncRNA HMMR-AS1 promotes proliferation and metastasis of lung adenocarcinoma by regulating MiR-138/sirt6 axis. Aging 11: 3041-3054, 2019.

34. Giono LE, Resnick-Silverman L, Carvajal LA, St Clair S and Manfredi JJ: Mdm2 promotes Cdc25C protein degradation and delays cell cycle progression through the $\mathrm{G} 2 / \mathrm{M}$ phase. Oncogene 36: 6762-6773, 2017.

35. Grey W, Ivey A, Milne TA, Haferlach T, Grimwade D, Uhlmann F, Voisset $\mathrm{E}$ and $\mathrm{Yu}$ V: The Cks1/Cks2 axis fine-tunes Mll1 expression and is crucial for MLL-rearranged leukaemia cell viability. Biochim Biophys Acta Mol Cell Res 1865: 105-116, 2018.

36. Liu L, Yang Y, Liu S, Tao T, Cai J, Wu J, Guan H, Zhu X, $\mathrm{He} \mathrm{Z}$, Li J, et al: EGF-induced nuclear localization of SHCBP1 activates $\beta$-catenin signaling and promotes cancer progression. Oncogene 38: 747-764, 2019. 
37. Zhang G, Yu Z, Fu S, Lv C, Dong Q, Fu C, Kong C and Zeng Y: ERCC6L that is up-regulated in high grade of renal cell carcinoma enhances cell viability in vitro and promotes tumor growth in vivo potentially through modulating MAPK signalling pathway. Cancer Gene Ther 26: 323-333, 2018

38. Hannabuss J, Lera-Ramirez M, Cade NI, Fourniol FJ, Nédélec F and Surrey T: Self-organization of minimal anaphase spindle midzone bundles. Curr Biol 29: 2120-2130.e7, 2019.

39. Breznau EB, Semack AC, Higashi T and Miller AL: MgcRacGAP restricts active RhoA at the cytokinetic furrow and both RhoA and Rac1 at cell-cell junctions in epithelial cells. Mol Biol Cell 26: 2439-2455, 2015.

40. Mack GJ and Compton DA: Analysis of mitotic microtubule-associated proteins using mass spectrometry identifies astrin, a spindle-associated protein. Proc Natl Acad Sci USA 98 14434-14439, 2001.

41. Gruber J, Harborth J, Schnabel J, Weber K and Hatzfeld M: The mitotic-spindle-associated protein astrin is essential for progression through mitosis. J Cell Sci 115: 4053-4059, 2002.

42. Maxwell CA, Benítez J, Gómez-Baldó L, Osorio A, Bonifaci N, Fernández-Ramires R, Costes SV, Guinó E, Chen H, Evans GJR, et al: Interplay between BRCA1 and RHAMM regulates epithelial apicobasal polarization and may influence risk of breast cancer. PLoS Biol 9: e1001199, 2011.

43. Cho YC, Park JE, Park BC, Kim JH, Jeong DG, Park SG and Cho S: Cell cycle-dependent Cdc25C phosphatase determines cell survival by regulating apoptosis signal-regulating kinase 1 . Cell Death Differ 22: 1605-1617, 2015.

44. Frontini M, Kukalev A, Leo E, Ng YM, Cervantes M, Cheng CW Holic R, Dormann D, Tse E, Pommier Y and Yu V: The CDK subunit CKS2 counteracts CKS1 to control cyclin A/CDK2 activity in maintaining replicative fidelity and neurodevelopment. Dev Cell 23: 356-370, 2012.
45. Schmandt R, Liu SK and McGlade CJ: Cloning and characterization of mPAL, a novel Shc SH2 domain-binding protein expressed in proliferating cells. Oncogene 18: 1867-1879, 1999.

46. Baumann C, Körner R, Hofmann K and Nigg EA: PICH, a centromere-associated SNF2 family ATPase, is regulated by Plk1 and required for the spindle checkpoint. Cell 128: 101-114, 2007.

47. Shen Y, Sherman JW, Chen X and Wang R: Phosphorylation of CDC25C by AMP-activated protein kinase mediates a metabolic checkpoint during cell-cycle G2/M-phase transition. J Biol Chem 293: 5185-5199, 2018.

48. Poser E, Caous R, Gruneberg U and Barr FA: Aurora A promotes chromosome congression by activating the condensin-dependent pool of KIF4A. J Cell Biol 219: e201905194, 2019.

49. Yen TJ: Polo delivers a PICH to the kinetochore. Cell 128: 20-21, 2007.

50. Spruck CH, de Miguel MP, Smith AP, Ryan A, Stein P, Schultz RM, Lincoln AJ, Donovan PJ and Reed SI: Requirement of Cks2 for the first metaphase/anaphase transition of mammalian meiosis. Science 300: 647-650, 2003.

51. Zhang T, Gong Y, Meng H, Li C and Xue L: Symphony of epigenetic and metabolic regulation-interaction between the histone methyltransferase EZH 2 and metabolism of tumor. Clin Epigenetics 12: 72, 2020.

This work is licensed under a Creative Commons Attribution-NonCommercial-NoDerivatives 4.0 International (CC BY-NC-ND 4.0) License. 